\title{
Contribution à l'étude de la vulnérabilité des terres de cultures de la région de Thiès (Sénégal) à l'aide de la télédétection et des systèmes d'information géographique (SIG)
}

Amadou SALL ${ }^{1}$, Assize TOURE ${ }^{1}$, Alioune KANE ${ }^{2}$, Awa Niang Fall ${ }^{2}$

${ }^{1}$ Centre de Suivi Écologique, Rue Léon Gontran Damas, Fann-Résidence, BP 15532, Email : amadou.sall@,cse.sn ; assize@,cse.sn;

2Département de Géographie, Université Cheikh Anta Diop de Dakear (UCAD), Email ; alioune.kane@ucad.edu.sn; awa10.fall@ucad.edu.sn

Correspondant email : amadou.sall@,cse.sn; amadou.sall@gmail.com Cél : (+221) 775507671

Mots clés : occupation du sol, étude multidate, modélisation, terres arables, Thiès

Keywords: land cover, dynamics, modeling, cultivated land, Thiès

Publication date 31/07/2019, http://www.m.elewa.org/JAPS;

\section{RESUME}

L'objectif de cette étude est d'établir à partir de la télédétection et des SIG, la dynamique spatio-temporelle des terres de cultures et d'explorer les futurs possibles de l'occupation du sol dans trois communes rurales de la région de Thiès (Fandène, Notto Diobass et Taiba Ndiaye). Une classification multidate des images landsat (1988, 2002 et 2014) a permis de quantifier les changements d'occupation des terres. Les résultats montrent que les zones de culture de Fandène sont passées entre 1988 et 2014 de $62 \%$ à $52 \%$ de la superficie totale de la commune. A l'opposée la commune de Taiba Ndiaye connait une expansion des zones de culture entre ces deux dates. Les changements enregistrés à Notto sont négligeables. Les simulations, faites sur la base des probabilités pour que la valeur d'une cellule i reste inchangée ou prenne la valeur d'une autre cellule $j$ à l'horizon 2035, révèlent que les terres de culture de Fandène ont $69 \%$ de probabilité d'évoluer vers d'autres classes d'occupation du sol.

\begin{abstract}
The objective of this study is to quantify from remote sensing and GIS the spatio temporal dynamics of cultivated land and explore possible futures of land use in three rural municipalies of Thies (Fandene, Notto Diobass, and Taiba Ndiaye). A multidate classification Landsat images (1988, 2002 et 2014) was used to quantify change in land cover. The results show that between 1988 and 2014 Fandene cropping areas have passed from 62\% to $52 \%$ of the total area. At the opposite the commune of Taiba Ndiaye has known an expansion of cropping areas between these two dates. Minor changes are noted in Notto district. Simulations carried out on the basis of probabilities for a unit $i$ to stay in the same cell or to be converted to another unit $j$ in 2035 , reveals that the probability for a cultivated land unit to be transformed into a another land cover category is high in Fandene (69 \%).
\end{abstract}




\section{INTRODUCTION}

L'Afrique de l'ouest, et en particulier le Sahel sont reconnus comme des régions très vulnérables au changement climatique et à la dégradation des terres avec comme principale menace une modification de l'occupation du sol. L'intensité de ces modifications, diffère en fonction du climat, des systèmes de production, de l'efficacité des solutions de conservation et de gestion des terres. Actuellement, les Nations Unies ont émis un certain nombre de recommandations aux pays membres pour le suivi environnemental, dont l'utilisation de la technologie géo-spatiale (Thomas et al. 2012). La communauté scientifique dispose maintenant de moyens efficaces pour recueillir et analyser des données et informations géospatiales appropriées grâce à l'exploitation de l'imagerie satellitale, qui permet de révéler l'état des terres et mettre en évidence les liens entre les différentes régions de la planète. L'image satellitale est sans doute le modèle visuel le plus fidèle, le plus riche de l'espace géographique. Elle est la source d'informations abondantes et précises sur l'occupation du sol et sur les phénomènes qui se déroulent à la surface de la terre. Tout autour du globe terrestre, les clôtures des exploitations agricoles et les différents types d'utilisation et d'occupation des terres sont visibles depuis l'espace. Les données satellites sont donc d'un grand apport pour les études de détection des changements d'utilisation et d'occupation du sol ( Heiskanen et al. 2017). La télédétection a permis d'étudier les changements d'occupation du sol en moins de temps, à faible coût et avec une meilleure précision (Corgne et al., 2016). Elle est couplée avec les SIG qui fournissent une plate-forme appropriée pour l'analyse et la mise à jour des données (Goodchild and Barbara, 2009). La littérature est foisonnante de cas d'étude présentant de nombreuses expériences sur les changements d'occupation du sol qui affectent la biodiversité, la disponibilité de l'eau, les pâturages et les zones de culture (Faye et al. 2016 ; Soro et al. 2014). Néanmoins, la caractérisation des principaux types de couverture terrestre et d'utilisation des terres, et leur suivi dans un paysage agricole représentent toujours un défi scientifique, puisqu'elles sont caractérisées par une "impermanence temporelle et spatiale" à différentes échelles allant du long terme au court terme et du niveau régional au niveau local; ce qui constitue un obstacle à la compréhension de leur fonctionnement. Dans ce contexte changeant et incertain, une bonne connaissance des variations spatio-temporelles de la couverture et de l'utilisation du sol représente un élément clé, tant pour la communauté scientifique que pour les acteurs du secteur agricole, qui veulent adresser la problématique de la dégradation des terres agricoles. Après plusieurs décennies d'application de la télédétection au Sahel, les capacités sont encore limitées pour un suivi rigoureux et cohérent de l'utilisation et occupation des sols. Le Centre de Suivi Ecologique sénégalais (CSE) et le Centre Régional d'AGRHYMET (CRA) sont parmi les rares exemples d'institutions africaines à consentir des efforts à long terme pour quantifier la dynamique des terres au Sahel, en raison des ressources financières limitées. Encore que ces analyses sont menées au travers des études de cas. Ainsi, de nombreuses études de terrain sont limitées à quelques pays sahéliens. Des programmes tels que GLADA (évaluation globale de la dégradation et de l'amélioration des terres) ont utilisé la télédétection afin de quantifier la réduction des terres agricoles disponibles dans les zones arides d'Afrique exacerbée par la fragilité des sols et la dégradation rapide des terres (Mbow et al., 2015). Dans la région de Thiès, plusieurs études en SIG et télédétection abordant diverses thématiques y ont été réalisées (Desthieux 2000, Repetti 2003, Sansonnens 2005, Sall et Dieye 2011). Cependant, ces études n'ont pas permis de qualifier et de quantifier sur plusieurs périodes les modes d'occupation et d'usages du sol sur l'ensemble de notre zone d'étude et de simuler 
l'occupation du sol dans le futur. Ce travail de recherche tente de combler ce gap. Il vise à caractériser les modes d'occupation et d'utilisation du sol, notamment agricole, à analyser leur dynamique spatio-temporelle sur

\section{MATERIEL ET METHODES}

3.1 Présentation de la zone d'étude: $\mathrm{La}$ zone d'étude se situe dans la région de Thiès à l'ouest du Sénégal. Elle est à cheval entre une partie littorale située dans les Niayes (Taïba Ndiaye) et une partie continentale (Fandène et Notto) (figure 1). La région est constituée d'un relief relativement plat, excepté le plateau de Thiès (105 m d'altitude), le massif de Diass (90 m d'altitude) et la cuvette de Thiès (128 m d'altitude). Elle fait partie de la Zone Agricole du Centre-Ouest (ZACO) connue sous le nom de Bassin arachidier. La région se situe dans une zone de transition soumise à l'influence des alizés maritimes et de l'harmattan. Le climat est de type soudano sahélien. Avant le début de la grande sécheresse des années 1970, la normale une période de 26 années d'observation (19882014) et à procéder à des simulations de l'évolution de l'occupation des terres à l'horizon 2035.

pluviométrique était de $579 \mathrm{~mm}$. Elle a fortement chuté pour se situer actuellement à $492 \mathrm{~mm}$ soit une baisse de $150 \mathrm{~mm}$ (ANACIM, 2009). Les principaux types de sols rencontrés dans la zone sont les sols ferrugineux lessivés (Dior), les sols ferrugineux non lessivés (Deck), les sols hydromorphes et les sols peu évolués. La végétation est composée de savane arbustive dégradée parsemée de peuplements monospéfiques d'Acacia seyal, d'Andnsonia digitata (Baobab), d'un parc à Faidherbia albida et de Borassus aethiopum (palmiers rôniers). L'agriculture sous pluie est la principale activité à laquelle s'adonne la quasitotalité de la population de la région. La forte pression sur les terres et l'état de saturation qui a suivi, ont modifié le système de production.

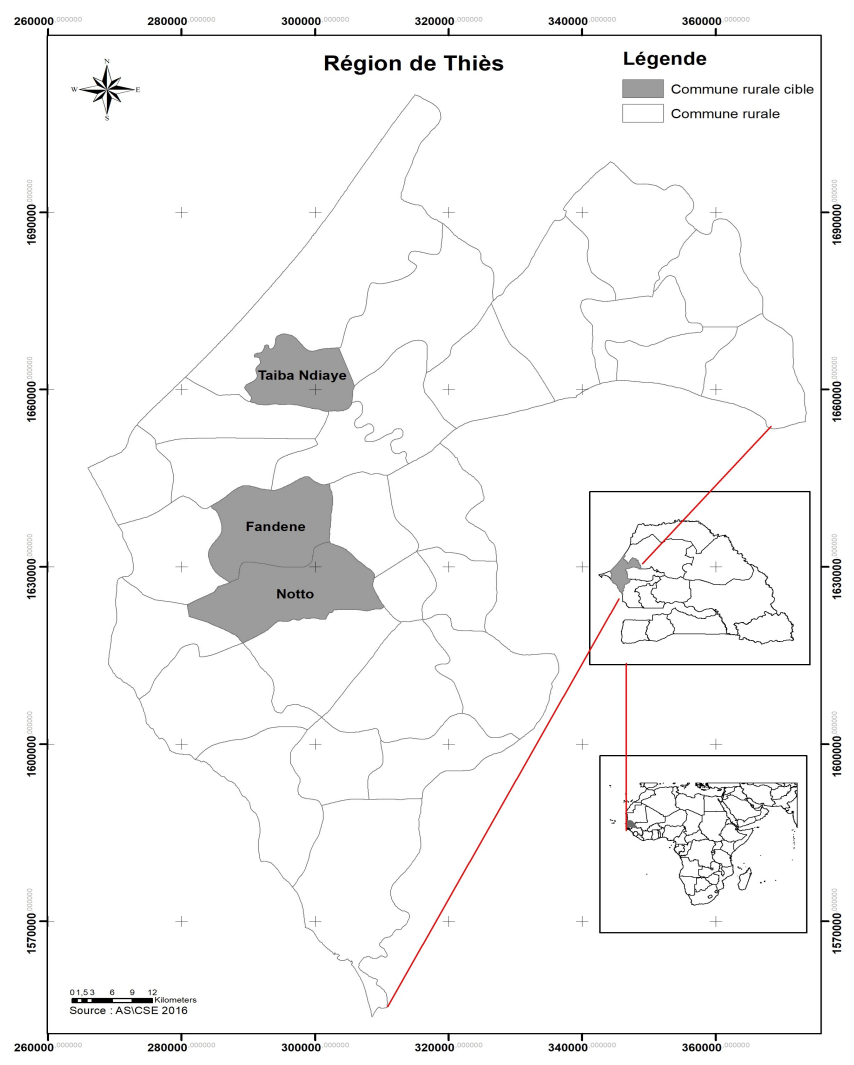

Figure 1 : Carte de la zone d'étude 


\subsection{Données et processus de traitement}

3.2.1 Données : Les images satellite spécifiques utilisées ont été Landsat ETM de 1988 et 2002, Land Imager (OLI) pour 2014. Avec une résolution de $30 \mathrm{~m}$, les images Landsat sont suffisamment détaillées pour permettre la cartographie et l'évaluation de nombreux types de changements au sein des paysages, en particulier l'expansion ou la régression de terres agricoles et des habitations, ainsi que la fragmentation des forêts et des savanes. La régularité et la continuité de l'imagerie Landsat permet de faire des observations objectives en matière de changements dans l'occupation des terres entre 1988 et 2014. De plus, toutes les images d'archive Landsat sont maintenant disponibles gratuitement. Ce qui représente une masse critique d'informations pour identifier et surveiller les changements environnementaux (Chander, 2009; Bastawesy 2014). Nous avons travaillé avec les images prises durant la saison humide (octobre, novembre, décembre). Les données satellites couvrant la zone d'étude ont été obtenues à partir des sites du global land cover facility (GLCF) (http://glcfapp.glcf.umd.edu:8080/esdi/) et de Earth Explorer (http:// earthexplorer.usgs.gov/).

\subsubsection{Procédures de classification et de} modélisation

3.2.2.1 Procédures de classification : Les cartes d'occupation du sol peuvent être crées par une interprétation visuelle des images satellites (en particulier avec des données de hautes résolution spatiale) ou par des méthodes de classification supervisée ou non supervisée. Dans le cas de l'interprétation visuelle des images, les pixels d'un type spécifique d'occupation du sol sont identifiés et délimités par un spécialiste en SIG (Lillesand et al., 2014). Cependant cette procédure est longue et couteuse. C'est pour cette raison que nous avons opté pour la classification supervisée. La connaissance de la zone d'étude et des catégories thématiques (tableau 1) présentes sur le secteur d'étude a aussi guidé le choix en faveur de la classification supervisée. La figure 2 donne un aperçu global simplifiant les étapes de la procédure de classification. L'algorithme Maximum de Vraisemblance (Maximum Likelihood) a été choisi pour la classification des images. Le maximum de vraisemblance fait partie des classificateurs dits paramétriques. L'intérêt majeur des approches paramétriques est de faire intervenir les informations relatives à la classe d'appartenance du pixel sous forme de probabilité. Nous avons procédé par échantillonnage dans une ou plusieurs zones réputées être occupées par une catégorie thématique connue, nous avons sélectionné quelques portions de surfaces représentatives et avons demandé à l'algorithme d'extraire la distribution des luminances dans chaque bande. Ces informations constituent la signature spectrale de l'échantillon (Caloz et Collet, 2001). Dans la phase suivante, le logiciel Idrisi a regroupé en classes spectrales tous les pixels présentant des propriétés spectrales similaires à celles des échantillons. Le modèle LCM (Land Change Modeler) qui est un module intégré dans le logiciel IDRISI est utilisé pour quantifier les changements, les pertes et gains pour chaque catégorie d'occupation du sol entre les différentes dates. 


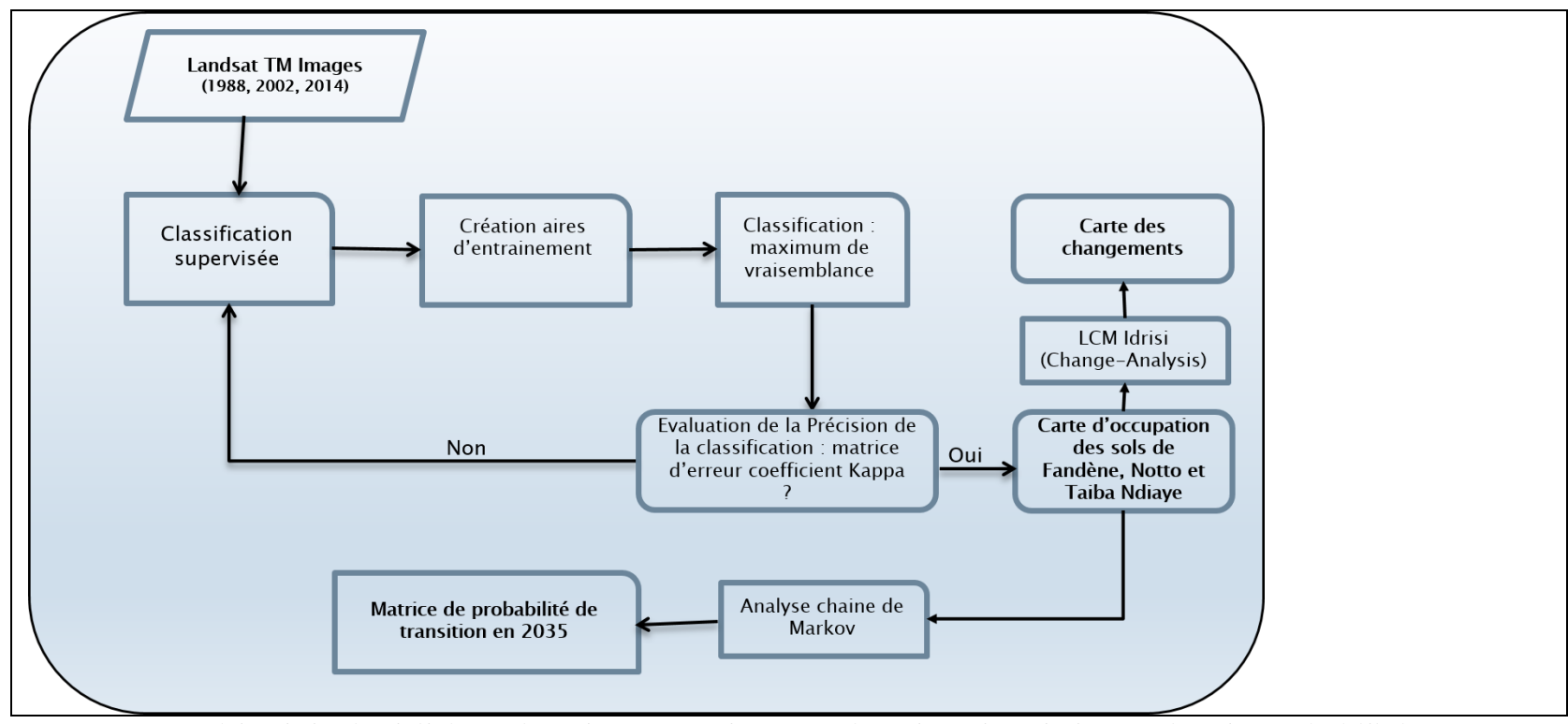

Figure 2 : Méthodologie d'élaboration des cartes d'occupation du sol et de la matrice de probabilité

3.2.2.2 Observation de terrain : La vérification des classes d'occupation du sol de la carte de 2014 a permis une évaluation de la qualité des résultats obtenus suivie de relevés sur l'ensemble de la zone d'étude (figure 3). Cette mission s'est déroulée entre 2010 et 2016 mais à la même date d'acquisition des images. L'indice de Kappa, utilisé pour apprécier la précision de la classification, est de $79 \%$. D'après
Pontius (2000) dans une étude de l'occupation du sol, lorsque l'indice de Kappa évalué dans les opérations de classification est compris entre 50 et $75 \%$, la classification adoptée est valable et les résultats peuvent être judicieusement utilisés. Le recours à des personnes ressources a permis de mieux comprendre la dynamique de la zone d'étude au cours de la période 1988-2014.

Tableau 1: Unités d'occupation du sol

\begin{tabular}{l|l|l}
\hline Code & Nom de la classe & Description \\
\hline ZA & Zone artificialisée & Résidentiel, industriel, routes, commercial \\
\hline ZCP & Zone de culture pluviale & L'agriculture dépendant de la pluie \\
\hline ZCMA & $\begin{array}{l}\text { Zone de culture maraichère } \\
\text { et arboriculture }\end{array}$ & $\begin{array}{l}\text { Périmètre de culture maraichage dans les bas-fonds et } \\
\text { arboriculture }\end{array}$ \\
\hline ZSA & $\begin{array}{l}\text { Zone de savane arborée ou } \\
\text { habitats naturels }\end{array}$ & Mosaïque de formations végétales parsemée d'arbres isolés \\
\hline ZD & Zone dénudée & Zones cuirassées, sol nu, ravines \\
\hline
\end{tabular}



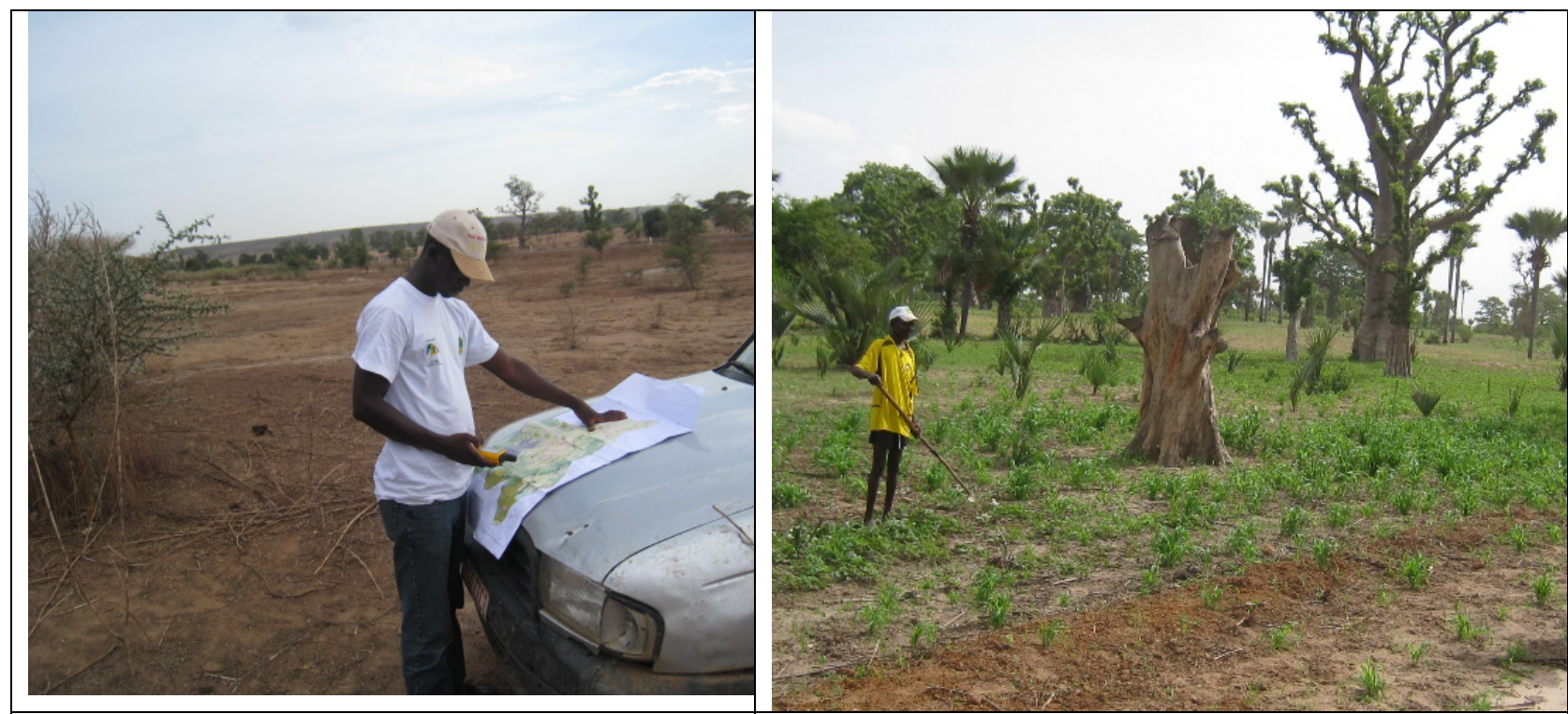

Figure 3 : Observations de terrain (crédit photo A. Sall)

3.2.2.3 Procédures de modélisation : Cette étape de la démarche nous conduit à prévoir les changements futurs, pour autant que le contexte considéré pour ces modèles soit encore pertinent dans le futur. Le type de modèle appliqué dans cette recherche est celui faisant appel aux chaines de Markov. Une séquence dans laquelle l'état à un moment donné est partiellement dépendant de l'état antérieur, est considérée comme une chaine de Markov. La méthode s'applique à décrire le comportement évolutif d'une seule observation sur la base d'une série temporelle. Il s'agit précisément d'évaluer la probabilité de transition d'un état à un autre, à l'aide de la matrice de probabilités de transition. Celle-ci fournit ainsi des informations sur la

\section{RESULTATS}

4.1 La répartition actuelle de l'occupation du sol: Pour rappel, les superficies de Fandène, Notto Diobass et Taiba Ndiaye sont respectivement d'environ $228 \mathrm{~km}^{2}, 255 \mathrm{~km} 2$ et $148 \mathrm{~km}$. Donc, 1\% de la superficie correspond à environ $2,2 \mathrm{~km}^{2}$ à Fandène, $2.5 \mathrm{~km} 2$ à Notto et 1,4 km2 à Taiba Ndiaye. Les zones de culture désignent à la fois les terres de culture pluviale et les milieux où sont pratiqués le maraichage et l'arboriculture. Les zones de culture qui occupent la plus grande partie de la zone d'étude, entre 50 et $70 \%$ de la superficie totale (fig. $4 \mathrm{~d}, 6 \mathrm{~d}$ et $8 \mathrm{~d}$ ), se répartissent autour des villages selon leur importance et vocation. Les cultures pluviales occupent $45,7 \%$ de la superficie de la probabilité de transition d'un état $\mathrm{A}$ à un état $\mathrm{B}$ en fonction de la probabilité d'apparition du premier état. Il s'agit donc d'une probabilité conditionnelle au passé (Antoni, 2006). Ce modèle markovien est présent dans différents logiciels de systèmes d'information géographique en mode image, notamment IDRISI (Eastman, 2012) utilisé ici pour analyser les probabilités de transition des différentes unités d'occupation du sol. L'étape d'analyse s'établit sur l'évolution de l'occupation du sol observé en 2014 ; sur cette base, le modèle propose l'état en 2035. En sus des zones agricoles, l'évolution des autres unités d'occupation du sol comme la zone artificialisée, la zone de savane et la zone dénudée, est mesurée.

commune de Fandène et sont globalement situées à l'est de la commune. Ces cultures occupent 56,7\% des terres de Notto Diobass et sont principalement localisées à l'est et aussi au sud-ouest de la commune. Enfin a Taiba Ndiaye les cultures pluviales sont presque reparties sur l'ensemble de la commune notamment dans le centre, le sud-est et l'extrême ouest. Les grandes cultures pluviales identifiées dans la zone d'étude sont essentiellement le mil, l'arachide, le niébé et le manioc. Le maraichage et l'arboriculture se superposent de manière générale au réseau hydrographique. Cette classe d'occupation du sol occupe $7,1 \%$ des terres à Fandène, $12,7 \%$ à Notto et $15,1 \%$ à Taiba Ndiaye. 
Les spéculations les plus importantes dans la zone sont l'oignon, le chou, la tomate, le piment et la laitue. Cependant on note une forte présence dans les champs de la carotte, du navet, de la pomme de terre et du haricot durant la campagne froide de Novembre à Mars. L'arboriculture est principalement constituée par les manguiers, les agrumes et l'exploitation des rôniers. Les zones dénudées localisées principalement au nord et au sud-ouest de Fandène, à l'ouest de Notto et au nord-est de Taiba Ndiaye couvrent respectivement $11,5 \% ; 2,6 \%$ et $5 \%$ de la superficie totale. La végétation naturelle est fortement représentée dans la partie ouest de Fandene et Notto abritant la forêt classée de Thiès, et au nord de Taiba Ndiaye. Elle est composée d'un tapis herbacé discontinu s'appauvrissant d'année en année et des espèces arborescentes arbustives assez variées composées essentiellement d'Acacia albida, de Guiera senegalensis, et dans une moindre mesure d'Adansonia digitata (baobab). La végétation naturelle couvre respectivement $22,1 \% ; 22,7 \%$ et $15,2 \%$ des communes de Fandène, Notto et Taiba Ndiaye. Les zones artificialisées sont surtout localisées à Fandène en raison de sa position géographique par rapport à la Ville de Thiès qu'elle ceinture. L'artificialisation concerne $13,2 \%$ des terres de Fandène, 5,3\% de la superficie de Notto et 6,1\% des terres de Taiba Ndiaye.

4.2 La dynamique de l'occupation du sol entre 1988 et 2014

4.2.1 Régression des zones de culture dans la commune de Fandène: Les résultats indiquent une absence de dynamique linéaire des changements d'occupation du sol. La figure 4 présente une synthèse des trois cartes d'occupation du sol et des statistiques issues de l'analyse diachronique. La commune de Fandène a enregistré au cours des 30 dernières années une diminution importante des zones de culture, qui occupent la plus grande partie de l'espace de la zone d'étude, au profit des zones artificialisées. Les zones de culture (pluviale et maraichage et arboriculture) représentaient en 1988 un peu plus de $60 \%$ des terres alors qu'en 2002, elles sont à moins de 50\% et couvrent à peine $53 \%$ des terres en 2014 (figure 4d). Le maraichage et l'arboriculture sont en déclin à Fandène $(10,9 \%$ en 1988 et $7.4 \%$ en 2014) qui est en grande partie bâtie sur une cuvette inondable qui permet la pratique du maraîchage comme culture d'appoint pendant la saison sèche. La concurrence imposée (en termes de qualité et de quantité) par les légumes issus de la zone des niayes limite sérieusement le développement de cette activité. La végétation naturelle, les zones de culture pluviale et les superficies dédiées au maraichage et à l'arboriculture ont respectivement connu une dynamique régressive de l'ordre de $-18 \% ;-47 \%$ et $-15 \%$ (figure 5 ). Les zones artificialisées et les zones dénudées enregistrent une augmentation de superficie de l'ordre de $61 \%$ et $50 \%$. De façon générale, on note que les taux d'occupation du sol des zones artificialisées et des zones dénudées ont augmenté entre 1988 et 2014. Cette augmentation s'est principalement opérée au détriment des zones de culture pluviale. 

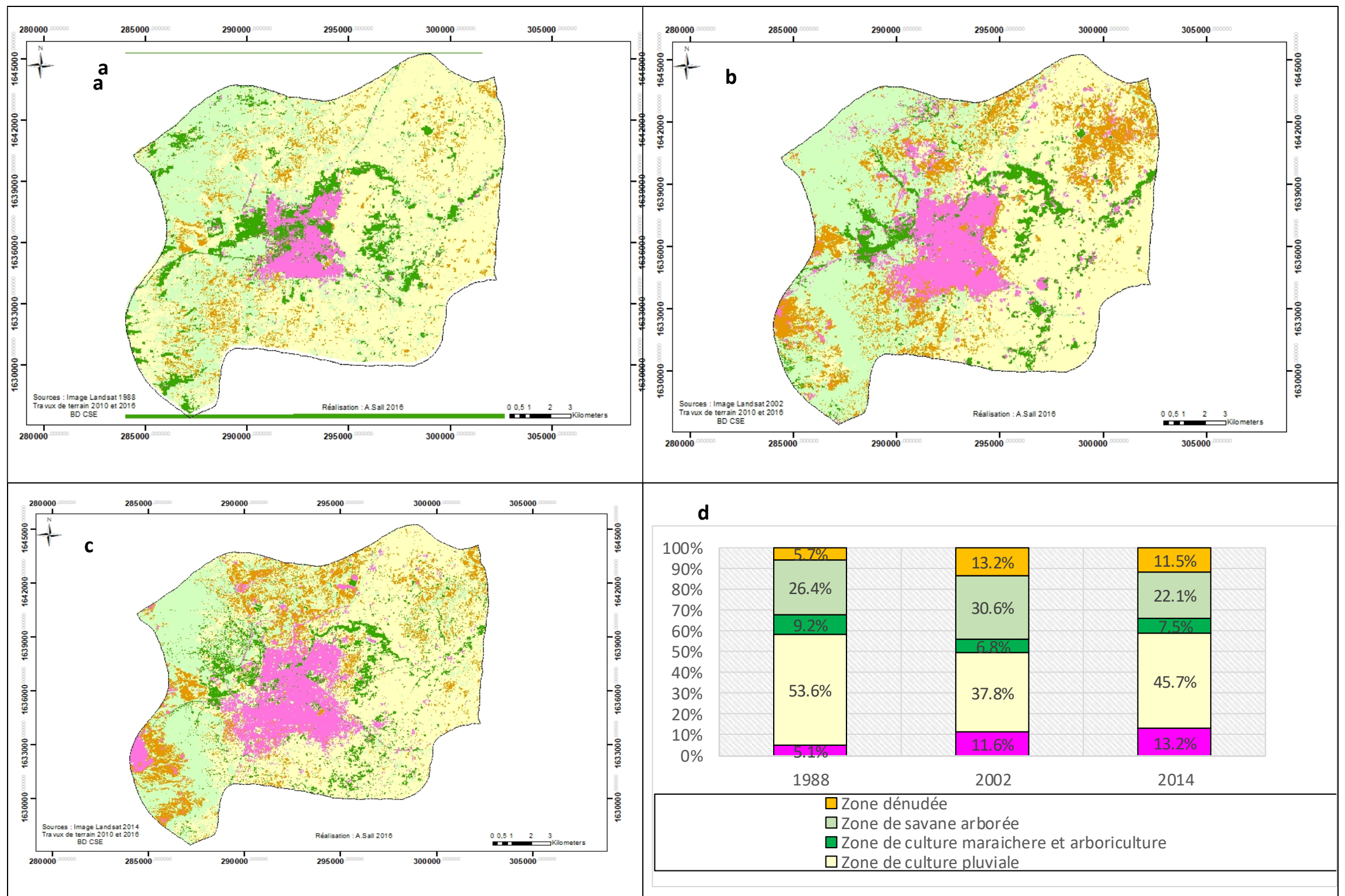

Figure 4 : Répartition des classes d'occupation du sol dans la commune de Fandène en a) 1988, b) 2002 et c) 2014. d) Evolution des superficies entre 1988, 2002 et 2014 


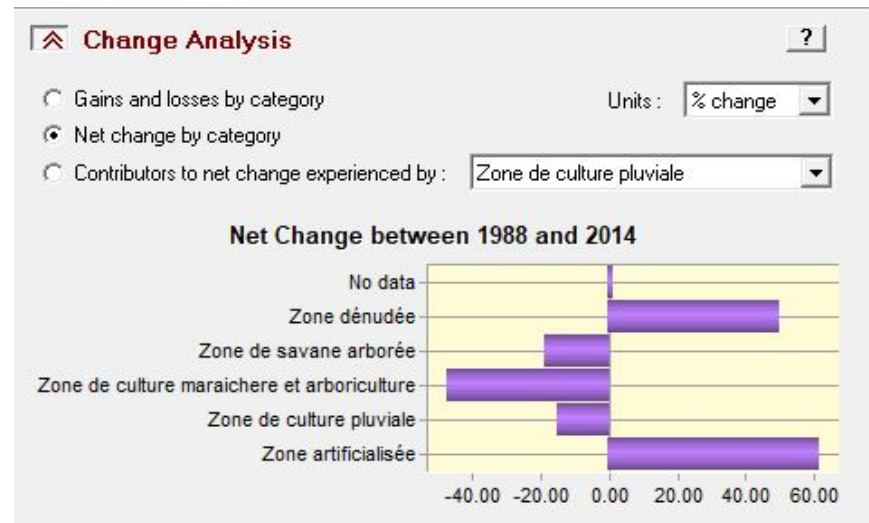

Figure 5 : Bilan par catégorie d'occupation du sol à Fandene entre 1988 et 2014, en pourcentage

\subsubsection{Quasi stabilité des zones de culture} dans la commune de Notto Diobass : L'examen de la figure 6 permet de constater qu'entre 1988 et 2014, les changements intervenus dans les modes d'occupation de l'espace sont négligeables. La plupart des zones sont restées à l'état initial. Les zones de culture enregistrent une régression de 3 points entre 1988 et 2014 . Sur la même période, la végétation naturelle est passée de $20 \%$ à $22,7 \%$ de la superficie. La figure $7 \mathrm{a}$ montre que les pertes et gains des formations végétales naturelles et les zones de maraichage et d'arboriculture tendent à s'équilibrer. L'essentiel de la dynamique spatiotemporelle consiste en un changement de localisation de ces classes. Ce qu'elles ont perdu à un endroit, elles l'ont gagné ailleurs. Quant aux zones de culture pluviale, elles gagnent 3431 ha et perdent en retour 4282 ha. Elles ont régressé de 851 ha (figure $7 \mathrm{~b}$ ) au profit des zones de culture maraichère et arboriculture, des zones artificialisées, des formations végétales naturelles et des zones dénudées respectivement de 592 ha; 214 ha; 30 ha et 14 ha au cours de la période 1988-2014 (figure $7 c)$. 


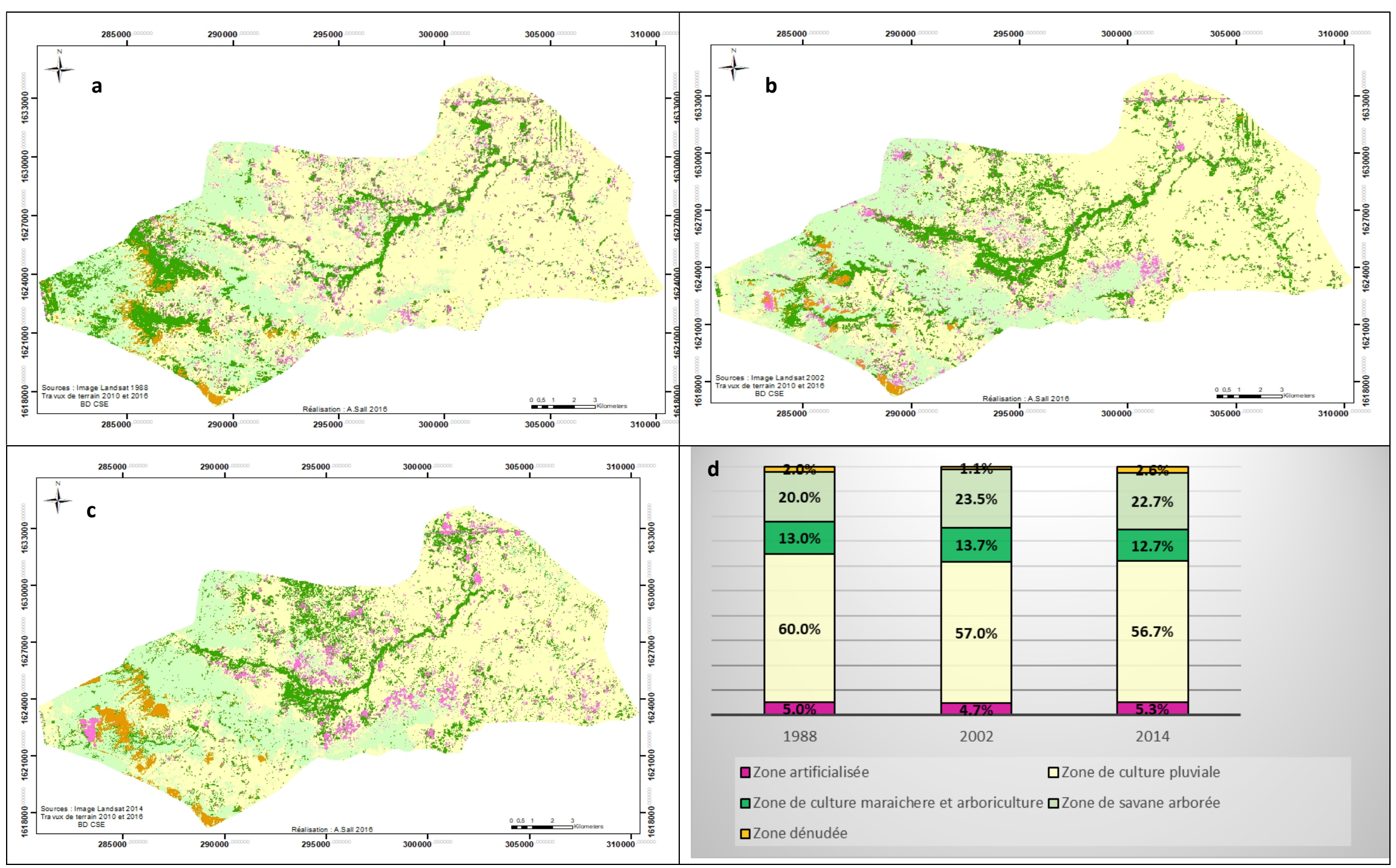

Figure 6 : Répartition des classes d'occupation du sol dans la commune de Notto Diobass en a) 1988, b) 2002 et c) 2014. d) Evolution des superficies entre 1988, 2002 et 2014 


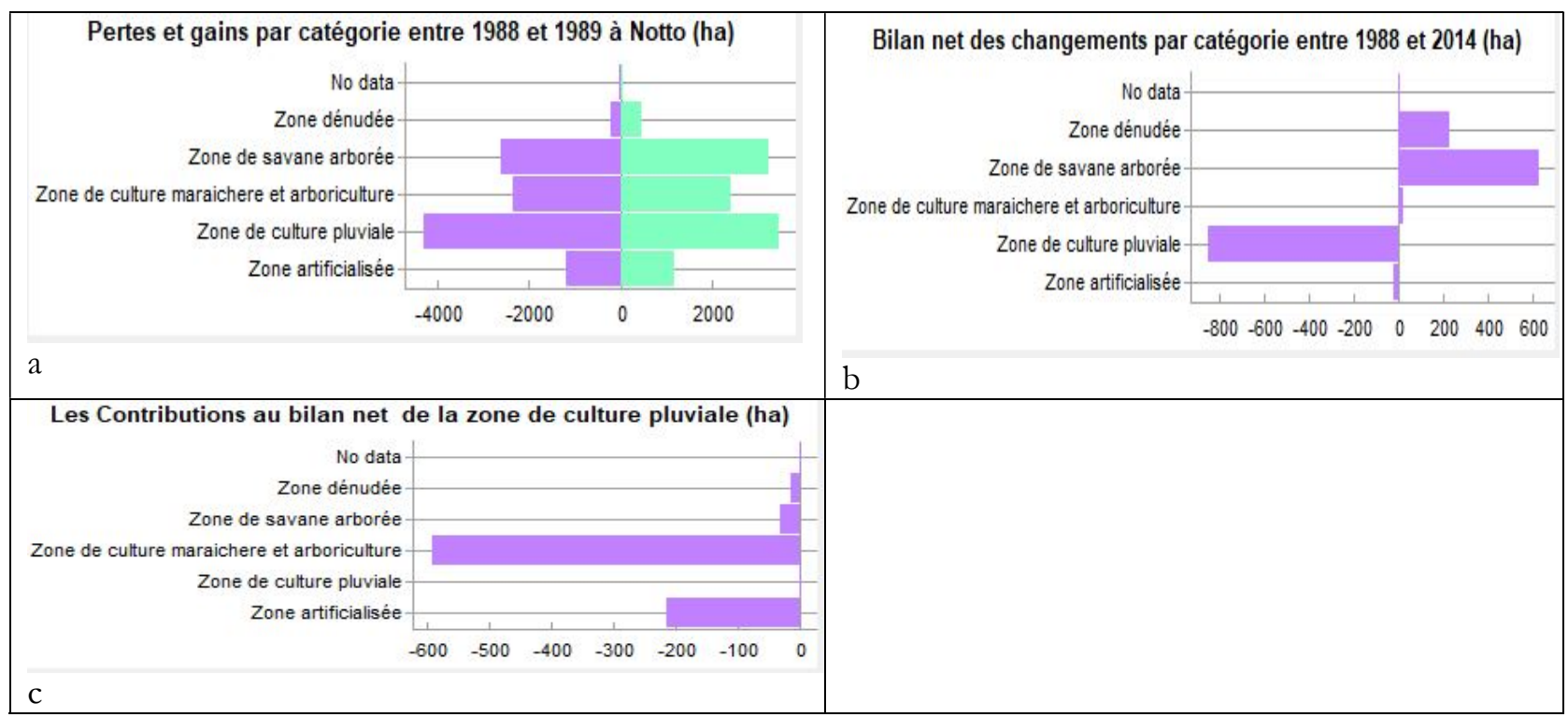

Figure 7 : Dynamique de l'occupation des terres à Notto entre 1988 et 2014, en hectare

\subsubsection{Expansion des terres de culture dans la} commune de Taiba Ndiaye: A Taiba Ndiaye entre 1988 et 2014, les zones de culture pluviale sont passées de $45 \%$ à $58,6 \%$ (figure $8 d$ ). La culture du manioc qui offre une alternative intéressante, face au déficit pluviométrique, peut expliquer la dynamique d'expansion des terres de culture pluviale. En effet, la plantation de manioc permet d'occuper les terres pendant au minimum les deux années de culture au lieu de les laisser sans exploitation. Les paysans l'expliquent par le fait que le manioc serait plus adapté aux sols riches en phosphate et en calcaire de la zone et à la faible fertilité des sols. Entre 2002 et 2014, on note une progression de la classe culture maraichère et arboriculture qui est passée de 7,9\% en 2002 à 15\% en 2014 quoique l'approfondissement de la nappe mette la ressource hydrique hors de portée de la plupart des maraîchers. L'arboriculture représente une solution à la crise climatique. L'augmentation de ces zones de culture s'est faite au détriment de la classe savane et végétation naturelle qui est passée de 36,8\% en 1988 à 15, 2\% en 2014 (figure 8d et 9). 


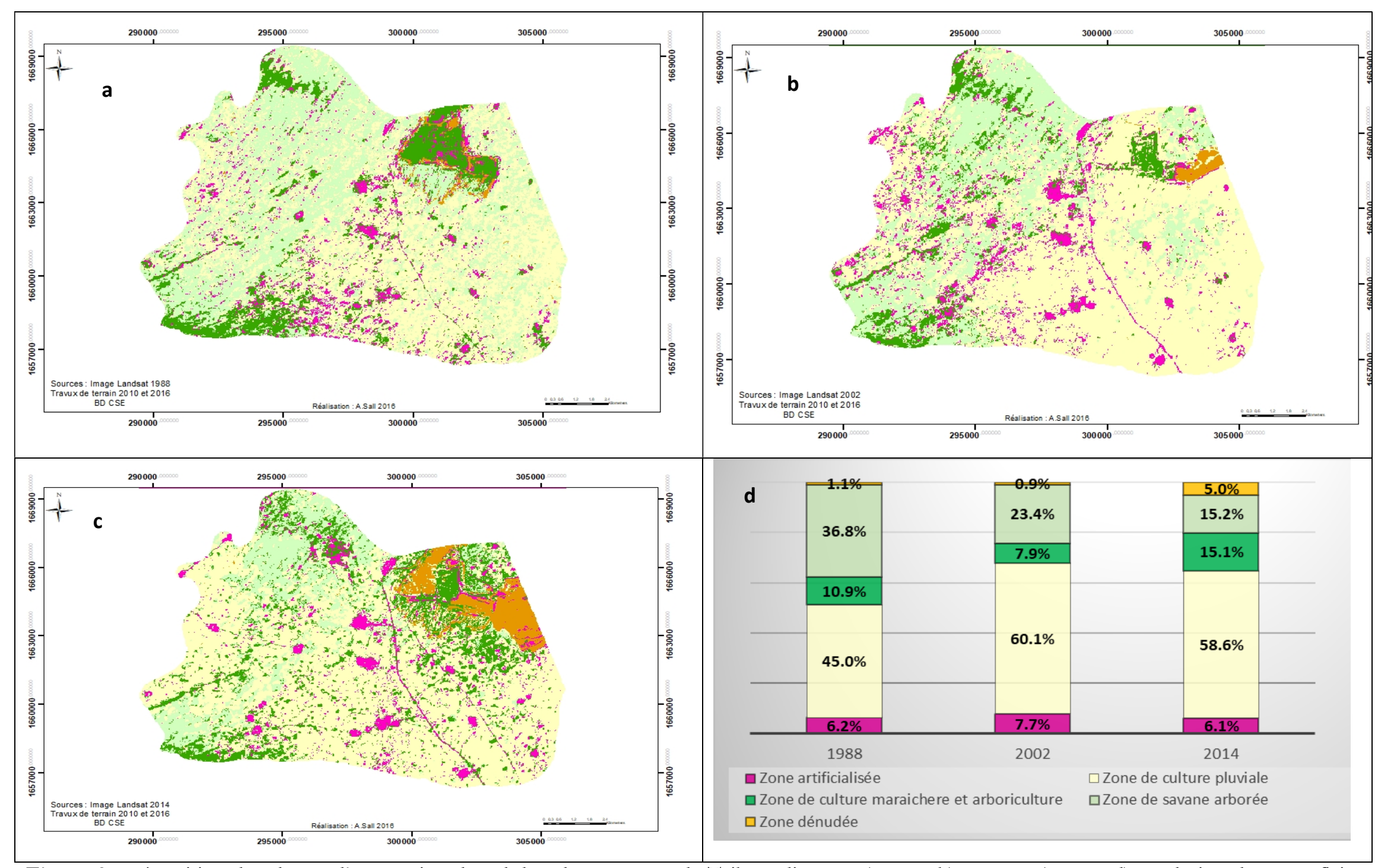

Figure 8 : Répartition des classes d'occupation du sol dans la commune de Taiba Ndiaye en a) 1988, b) 2002 et c) 2014. d) Evolution des superficies entre 1988, 2002 et 2014 

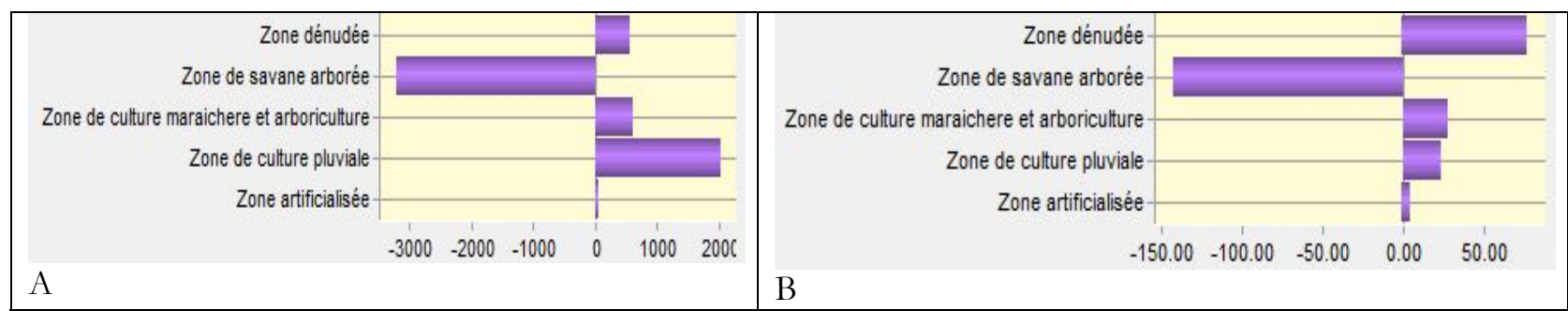

Figure 9 : Extension et régression des classes d'occupation du sol à Taiba Ndiaye entre 1988 et 2014, en hectare (A) et en pourcentage (B)

On observe deux grands processus dans le paysage de Fandène, Notto Diobass et Taiba Ndiaye en l'espace de 26 ans et qui menacent les terres de culture. Premièrement l'artificialisation du milieu qui s'est faite au détriment des terres de culture et deuxièmement le phénomène d'érosion observé dans les trois communes et la progression des surfaces dénudées qui en résulte.

4.3 Résultats de la matrice de transition de la chaine de Markov (2035) : Le module Markov a permis de calculer la matrice de probabilités de transition (Tableau 2). Elle donne pour chaque classe la probabilité de rester dans cette classe ou de se transformer en une autre à l'horizon 2035. L'analyse de cette projection révèle qu'à l'horizon 2035, les cultures pluviales présentent une probabilité de stabilité de $31 \%$ et $50,3 \%$ de devenir des zones artificialisées dans la commune de Fandène car le front d'urbanisation de la ville de Thiès continuera de menacer les terres de culture. A Notto et Taiba Ndiaye, contrairement à Fandène, les terres de culture pluviales seront assez stables avec plus de $60 \%$ de probabilité de rester dans la même catégorie $(63,2 \%$ pour Notto et $63,7 \%$ pour Taiba Ndiaye) et aussi avec de fortes probabilités de gagner des terres sur les autres classes malgré les incertitudes sur le climat et les pressions anthropiques. Les formations végétales naturelles de la commune de Taiba Ndiaye ont 51\% de probabilité d'évoluer vers des terres de culture pluviale. La tendance évolutive des espaces agricoles de Taiba Ndiaye provient de la place de choix accordée à l'agriculture dans l'économie de la zone.

Tableau 2: Matrice de probabilités de transition

\begin{tabular}{llllll}
\hline Fandène & $\mathbf{2 0 3 5}$ & \multicolumn{5}{l}{} \\
\hline 2014 & ZA & ZCP & ZCMA & ZSA & ZD \\
ZA & 0.7125 & 0.1550 & 0.0806 & 0.0244 & 0.0276 \\
ZCP & 0.5033 & 0.3135 & 0.0340 & 0.0573 & 0.0919 \\
ZCMA & 0.2878 & 0.3233 & 0.1564 & 0.1603 & 0.0721 \\
ZSA & 0.0775 & 0.2815 & 0.0708 & 0.4188 & 0.1514 \\
ZD & 0.0268 & 0.3443 & 0.0404 & 0.3427 & 0.2458 \\
\hline Notto & $\mathbf{2 0 3 5}$ & \multicolumn{5}{c}{} & \\
\hline 2014 & ZA & ZCP & ZCMA & ZSA & ZD \\
ZA & 0.2365 & 0.2686 & 0.2053 & 0.2301 & 0.0594 \\
ZCP & 0.0734 & 0.6958 & 0.1359 & 0.0912 & 0.0035 \\
ZCMA & 0.0797 & 0.3505 & 0.3727 & 0.1619 & 0.0351 \\
ZSA & 0.0252 & 0.2650 & 0.0612 & 0.5992 & 0.0492 \\
ZD & 0.0592 & 0.0340 & 0.1634 & 0.1774 & 0.5656 \\
\hline Taiba Ndiaye & $\mathbf{2 0 3 5}$ & \multicolumn{5}{c}{} & \\
\hline 2014 & ZA & ZCP & ZCMA & ZSA & ZD \\
ZA & 0.1843 & 0.5541 & 0.1675 & 0.0410 & 0.0514 \\
ZCP & 0.0588 & 0.6367 & 0.1422 & 0.1247 & 0.0360 \\
ZCMA & 0.1439 & 0.3008 & 0.2785 & 0.1120 & 0.1627 \\
ZSA & 0.0343 & 0.5194 & 0.1631 & 0.2506 & 0.0316 \\
ZD & 0.1421 & 0.1994 & 0.3175 & 0.0130 & 0.3276 \\
\hline
\end{tabular}




\section{DISCUSSION}

5.1 Pressions anthropiques sur les terres de culture : L'analyse des résultats obtenus à partir de l'analyse diachronique de l'occupation du sol entre 1988 et 2014, montre un empiètement du tissu urbain de la ville de Thiès sur le périmètre communal de Fandène. La plupart des lotissements ont été aménagés au sud et au sud-est de la Ville. Ceci parait difficilement compréhensible, étant donné que dans cette zone, se situent les meilleures terres agricoles et qu'au nord, le sol est beaucoup moins fertile. L'extension de l'urbanisation prive les villages phagocytés de leurs terres agricoles. En effet, le périmètre de la Ville de Thiès a été plusieurs fois étendu par décret entre 1954 et 1978, en intégrant d'anciens villages de la commune rurale de Fandène dont certains ont encore des caractéristiques très rurales. Les processus d'expropriation des habitants des villages pour développer les lotissements ont contribué à attiser les tensions entre la ville et la commune rurale. Il en découle la vente des terres de culture par les villageois ou paysans eux-mêmes (ce qui est illégal) causée par une peur certaine d'être exproprié, ou de perdre de ce qu'ils considèrent comme leur patrimoine (Ndiaye, 2013). Cette situation est observée au Kenya ou l'urbanisation a des impacts significatifs sur les terres de culture. Par exemple la production de café a chuté de plus de $50 \%$ en raison de la conversion de plantations de café du fait de projets immobiliers (UNEP, 2016). A l'opposé, cette pression anthropique sur les terres de culture peut également entrainer une expansion du front agricole principale cause $\mathrm{du}$ déboisement. Ce constat est corroborée par une étude menée par Faye et al. (2017) dans une autre partie du bassin arachidier. En effet, les sols cultivés, les savanes arbustives et les savanes boisées qui représentaient $31 \%, 27 \%$ et $40 \%$ des superficies du bassin en 1986 sont passées à $62 \%, 21 \%$ et $12 \%$ en 2016. Ces pertes en terres arables combinées à la croissance démographique et à la mécanisation de l'agriculture ont entrainé une intensification de l'utilisation des terres avec la réduction, voire la disparition de la jachère et l'extension des cultures dans des zones fragiles, un défrichement massif, la mise en culture des bas-fonds et une accélération du ruissellement (Ba, 2018). Cette dégradation des états de surface fait que l'érosion hydrique entraine dans certains endroits du Sénégal des pertes en terres proche de 1tonne/ha/an (Albergel et al., 1990).
5.2 Erosion des sols : Le second fait marquant de nos résultats est l'importance de l'érosion. Les sols sont soumis pendant une longue période à des phases poussées de dessiccation, avec peu ou pas de couverture végétale. Desthieux (2000) a identifié la présence de sols squelettiques d'érosion (lithosols) qui se manifestent principalement sur les versants des collines de Thiès où la pente est d'intensité suffisante (4 à $7 \%$ ) pour provoquer une érosion en ravine et en nappe importante. Cissokho (2011) a montré que le niveau de vulnérabilité et de susceptibilité à l'érosion éolienne dans la région de Thiès est important. On note parfois de légères dynamiques d'amélioration. Mais ces dynamiques ne sont pas pérennes, elles fluctuent en fonction des caractéristiques des saisons. Ces actions érosives sont des phénomènes très inquiétants de par leur rapidité et expansion. L'entrainement de particules sableuses par le vent et le ruissellement provoquent, sur les sols ferrugineux tropicaux lessivés, l'affleurement de la cuirasse. Ces sols devenus rocailleux sont alors stériles et entrainent des pertes irréversibles en terres cultivables. A terme, si aucune mesure de lutte n'est entreprise, toute la partie de notre zone d'étude située sur les sols ferrugineux tropicaux sur cuirasse risque d'être intégralement cuirassée. Cette dégradation des terres de culture est observée dans le bassin arachidier où les sols deviennent de plus en plus pauvres (FAO/CSE, 2003) et très vulnérables à l'érosion avec la destruction du couvert végétal. D’après Lo et Sow (2017), les terres arables dégradées par type de contrainte dans le Bassin arachidier se présentent ainsi : salinisation (200 $000 \mathrm{ha}$ ), érosion hydrique (900 $000 \mathrm{ha}$ ), érosion éolienne (50 $000 \mathrm{ha}$ ). On estime qu'au cours des 40 dernières années, près d'un tiers des terres arables du monde ont disparu à cause de l'érosion et continuent de disparaitre à raison de plus de 10 millions d'hectares par an (Pimentel, 2006). En Afrique, les deux tiers des terres sont déjà plus ou moins dégradées et que cette dégradation touche au moins 485 millions de personnes, soit $65 \%$ de la population du continent (Fischer et al., 2012).

5.3 Tendances à l'horizon 2035: Il importe de noter que pour la technique de simulation de l'occupation du sol à l'horizon 2035, les tendances sont envisagées avec l'hypothèse que les facteurs potentiels et les variables qui influenceront la dynamique du paysage, garderont la même emprise que dans celle de la période de 1988 à 2014. Au vu 
des résultats de la matrice de probabilité, il est attendu une confirmation des tendances générales de la dynamique des terres agricoles observée à Fandène (régression), Taiba Ndiaye (extension) et Notto (stabilité). La perspective entrevue à Taiba Ndiaye et dans une moindre mesure à Notto Diobass est identique aux résultats publiés par l'Institut national de la recherche agronomique (INRA) et le Centre de coopération internationale en recherche agronomique pour le développement (CIRAD) cités par Lazarev (2009), qui montrent la possibilité d'expansion de la frontière agricole, grâce aux réserves en terres de certains grands pays (essentiellement en Amérique latine et en Afrique subsaharienne). Ces institutions entrevoient une

\section{CONCLUSION}

Ce travail a montré qu'avec des méthodes associant SIG et statistiques, il est possible d'enrichir les interprétations thématiques, la caractérisation et l'explication des dynamiques d'occupation du sol notamment les phénomènes de régression et d'extension des terres agricoles. L'analyse des dynamiques a permis d'identifier et caractériser l'espace agricole des trois communes étudiées et sa déformation entre plusieurs dates mais aussi de construire une matrice de probabilité d'évolution du paysage agricole à l'horizon 2035 à l'aide de la

\section{BIBLIOGRAPHIE}

Albergel J, Diatta M, Juncker E, Pérez P, Ruelle P and Sène $\mathrm{M}$ : 1990. Méthodes pour améliorer l'infiltration et réduire le ruissellement: présentation du cas du SineSaloum (Sénégal). Bulletin-Réseau Erosion, (10), pp.68-85.

ANACIM : 2009. Analyse agroclimatique de la Zone InfoClim. $14 \mathrm{p}$.

Antoni JP : 2006. Calibrer un modèle d'évolution de l'occupation du sol urbain. L'exemple de Belfort. European Journal of Geography.

Ba T : 2018. Dynamique spatio-temporelle des écosystèmes du bassin versant du Ferlo (Nord-Sénégal). UCAD.

Bastawesy ME: 2014. Hydrological scenarios of the Renaissance Dam in Ethiopia and its hydro-environmental impact on the Nile downstream. Journal of Hydrologic Engineering 20(7).

Caloz R and Collet C : 2001. Précis de Télédétection. Volume III : Traitements progression de la surface cultivée mondiale de $39 \%$ entre 2000 et 2050 (soit 12 millions d'hectares nouveaux par an). Cette expansion horizontale permettrait la satisfaction des besoins alimentaires en 2050. En revanche, la perspective entrevue à Fandène risque d'être aggravée par le changement climatique. Lo et Sow (2017), ont établi qu’à l'horizon 2035 un déficit pluviométrique combiné à une hausse des températures et éventuellement à la survenue d'évènements extrêmes (vents violents, vague de chaleur ou de froid, averses produisant des inondations) pourrait entrainer une dégradation avancée des terres arables et engendrer une baisse sensible de la productivité de l'agriculture.

chaine de Markov. D'après les simulations et les projections effectuées, la vulnérabilité de l'espace agricole de Fandène va s'accentuer vers les horizons 2035. Bien que le modèle permette de déterminer le nombre de cellules de chaque catégorie d'occupation du sol dans le futur, en revanche, il ne dit rien sur leur localisation. L'approche pourrait donc être enrichie par une analyse plus approfondie d'automates cellulaires basées sur des chaines de Markov. Le travail présenté n'est qu'un maillon qui peut servir de base pour d'autres développements.

Numériques d'Images de Télédétection. Presses Universitaires du Québec.

Chander, G., Markham, B. L., and Helder DL: 2009. Summary of current radiometric calibration coefficients for Landsat MSS, TM, ETM+, and EO-1 ALI sensors. Remote sensing of environment (113(5)): 893-903.

Cissokho R : 2011. Développement d'un indice de vulnérabilité à l'érosion éolienne à partir d'images satellitales, dans le bassin arachidier du sénégal: cas de la région de Thiès. Thèse soutenue à l'Université de Montréal. $251 \mathrm{p}$.

Corgne S, Hubert-Moy L and Betbeder J : 2016. Monitoring of Agricultural Landscapes Using Remote Sensing Data. In: Land Surface Remote Sensing in Agriculture and Forest. Elsevier, pp. 221-247. DOI: 10.1016/B978-1-78548-103-1.50006-6.

Sansonnens C : 2005. Gestion intégrée des eaux de surface du bassin versant de Thiès (SN) - 
Proposition d'un plan de gestion Travail. Université de Fribourg. 145p

Desthieux G : 2000. Conception d'un système déinformation geographique. Ecole Polytechnique Fédérale de Lausanne (EPFL). $62 \mathrm{p}$.

Eastman JR : 2012. IDRISI Selva Manual. IDRISI Selva Manual. DOI: 10.1109/TGRS.2002.802519.

FAO/CSE : 2003. L'évaluation de la dégradation des terres au Sénégal. $62 \mathrm{p}$.

Faye V, Mbow C and Thiam A : 2016. Évolution de l'occupation et de l'utilisation du sol entre 1973 et 2010 dans la zone agropastorale du lac de Guiers (Sénégal). La revue électronique en sciences de l'environnement 16.

Faye W, Orange D, Kane A, Niang AF and Minea I : 2017. Impacts de la variabilité climatique et de la pression démographique sur les ressources en eau du bassin de thysse kaymore (saloum-senegal). Lucrările seminarului geografic "dimitrie cantemir (44): 109-124.

Fischer G, Hizsnyik E and Prieler S: 2012. Scarcity and abundance of land resources: competing uses and the shrinking land resource base. 58 p.

Goodchild MF and Barbara S: 2009. GIS and Cartography. 500-505.

Heiskanen J, Liu J et Valbuena R: 2017. Remote sensing approach for spatial planning of land management interventions in West African savannas. Journal of Arid Environments 140: 29-41. DOI: 10.1016/j.jaridenv.2016.12.006.

Lillesand T, Kiefer RW, and Chipman J: 2014. Remote sensing and image interpretation: John Wiley \& Sons.

Lazarev G : 2009. La gouvernance territoriale et ses enjeux pour la gestion des ressources naturelles: Des approches novatrices pour lutter contre la désertification et la dégradation des terres et des eaux. Document thématique, (3)

Lo H and Sow S : 2017. Contributions Déterminées au Niveau National (CDN): volet adapatation secteur de l'agriculture. Dakar. $51 \mathrm{p}$.

Mbow C, Brandt M, Ouedraogo I: 2015. What four decades of earth observation tell us about land degradation in the Sahel? Remote
Sensing 7(4): 4048-4067. DOI: $10.3390 /$ rs 70404048 .

Ndiaye S : 2013. Rapport sur: employabilité des jeunes et femmes à l'échelle de la ville de Thiès et de son bassin de vie». 122 p.

Pimentel D: 2006. Soil erosion: A food and environmental threat. Environment, Development and Sustainability. 8(1), 119137 DOI: $10.1007 / \mathrm{s} 10668-005-1262-8$.

Pontius JRG: 2000. Quantification error versus location error in compararison of categorical maps. Photogrammetric Engineering and remote Sensing, 66(8) : 1011-1016.

Repetti A : 2003. Un concept de monitoring participatif au service des villes en développement. Approche méthodologique et réalisation d'un observatoire urbain. Faculté environnement naturel, architectural et construit. Ecole Polytechnique Fédérale de Lausanne (EPFL). These troisieme cycle. 199 p.

Sall A and Dieye AM: 201. Adaptation aux impacts du changement climatique. In: (ed. CSE), Dakar, 2011, 400 p.

Soro G, Ahoussi E K, Kouadio E K, Soro TD, Oulare S, Saley M B and Biemi J : 2014. Apport de la télédétection à la cartographie de l'évolution spatio-temporelle de la dynamique de l'occupation du sol dans la région des Lacs (Centre de la Côte d'Ivoire). Afrique Science: Revue Internationale des Sciences et Technologie 10(3).

Thomas RJ., Akhtar-Schuster M and Stringer LC : 2012. Fertile ground? Options for a science-policy platform for land. Environmental Science \& Policy 16: 122 135.

UNEP : 2016. L'avenir de l'environnement mondial, GEO-6, évaluation régionale pour l'Afrique. Nairobi. 216 P. 